[Case report]

\title{
Late-diagnosed cesarean scar pregnancy resulting in unexpected placenta accreta spectrum necessitating hysterectomy
}

\author{
Nanao Suzuki ${ }^{1)}$, Hyo Kyozuka ${ }^{1)}$, Toma Fukuda ${ }^{1)}$, Tsuyoshi Murata ${ }^{1)}$, Aya Kanno $^{1)}$, Shun Yasuda ${ }^{1)}$, \\ Akiko Yamaguchi $^{1)}$, Rei Sekine ${ }^{2)}$, Akihito Hata ${ }^{3)}$ and Keiya Fujimori ${ }^{1)}$ \\ ${ }^{1)}$ Department of Obstetrics and Gynecology, School of Medicine, Fukushima Medical University, Fuku- \\ shima, Japan, ${ }^{2)}$ Department of the Diagnostic Pathology, School of Medicine, Fukushima Medical Uni- \\ versity, Fukushima, Japan, ${ }^{3)}$ Meiji Hospital, Fukushima, Japan
}

(Received July 2, 2020, accepted August 21, 2020)

\begin{abstract}
Cesarean scar pregnancy (CSP) is a rare complication involving the implantation of the gestational sac in a cesarean delivery scar. The authors report a case of unexpected placenta accreta spectrum (PAS) caused by late diagnosed CSP, necessitating emergent hysterectomy. A 28-year-old Japanese woman with two previous cesarean deliveries presented to our hospital at 11 weeks of gestation with abnormal transvaginal ultrasound findings obtained at another hospital; however, transabdominal ultrasound revealed that the fetus was already present in the uterine cavity at this time. At 28 weeks, there was no evidence of placenta previa. The woman developed preeclampsia at 29 weeks, and a cesarean section was conducted. Intraoperative findings confirmed PAS, and hysterectomy was conducted immediately.
\end{abstract}

Key words : cesarean scar pregnancy, placenta accreta spectrum, late diagnosis

Abbreviations : cesarean scar pregnancy : CSP, confidence interval : CI, placenta accreta spectrum : PAS, transvaginal ultrasound : TVUS

\section{Introduction}

Cesarean scar pregnancy (CSP) is a rare complication involving the implantation of the gestational sac in a cesarean delivery scar ${ }^{1)}$. Because CSP is rare, its outcome with expectant management is not well known.

Placenta accreta spectrum (PAS) is a significant obstetric complication that can cause massive and life-threatening bleeding. PAS is strongly associated with placenta previa ${ }^{2}$. However, PAS may occur unexpectedly in the absence of placenta previa and not be diagnosed until delivery, necessitating emergent hysterectomy. Here, we present the case of a woman with CSP who later developed PAS without placenta previa. She was first diagnosed with PAS at the time of delivery and underwent emergent hysterectomy.

\section{Case presentation}

A 28-year-old Japanese woman, para 2 (two previous cesarean sections), presented to our hospital at 11 weeks of gestation because of potential CSP. At 9 weeks, she had undergone transvaginal ultrasound (TVUS) at another hospital, which showed the gestational sac to be positioned low in the uterus in the area of her previous cesarean scar. However, when the patient first presented to our hospital, transabdominal ultrasound could not clearly confirm CSP because there was no evidence of placenta preva and the fetus and placenta had grown toward and presented in the uterine cavity. As CSP could be neither confirmed nor ruled out, we opted to care-

Corresponding author: Hyo Kyozuka E-mail : kyozuka@fmu.ac.jp

(일 The Fukushima Society of Medical Science. This article is licensed under a Creative Commons [Attribu-

tion-NonCommercial-ShareAlike 4.0 International] license.

https://creativecommons.org/licenses/by-nc-sa/4.0/ 
fully monitor the perinatal conditions. At 28 weeks, TVUS showed placental attachment on the anterior wall with a distance between the placental margin and intra OS of at least $77 \mathrm{~mm}$. Seeing no evidence of placenta previa, and we assumed PAS to be less likely (Fig. 1).

At 29 weeks of gestation, the woman was admitted because of preeclampsia. Magnesium sulfate was used to prevent seizures, and intramuscular steroid treatment was administered to stabilize fetal respiration after delivery. Despite strict perinatal management, the patient's preeclampsia became more severe, with increased proteinuria. This prompted an emergent cesarean section at 30 weeks of gestation. At laparotomy, we observed medusa head infiltration of the uterine surface by the placenta along with bulging of the uterine wall, indicating the presence of PAS (Fig. 2). A baby weighing 1,534 $\mathrm{g}$ (Apgar scores 5 at $1 \mathrm{~min}$ and 7 at $5 \mathrm{~min}$ ) was delivered through a transverse incision in the uterine fundus, and emergent cesarean hysterectomy

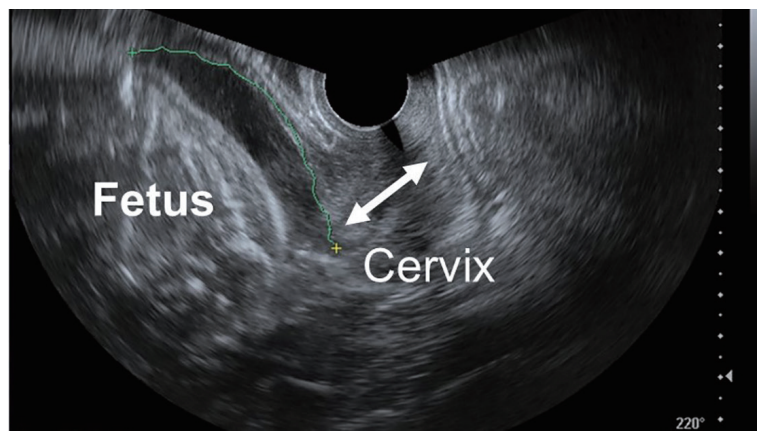

Fig. 1. Transvaginal ultrasound findings at 28 weeks. There is no evidence of placenta previa. The distance from the internal os to the margin of the placenta is $77.5 \mathrm{~mm}$.

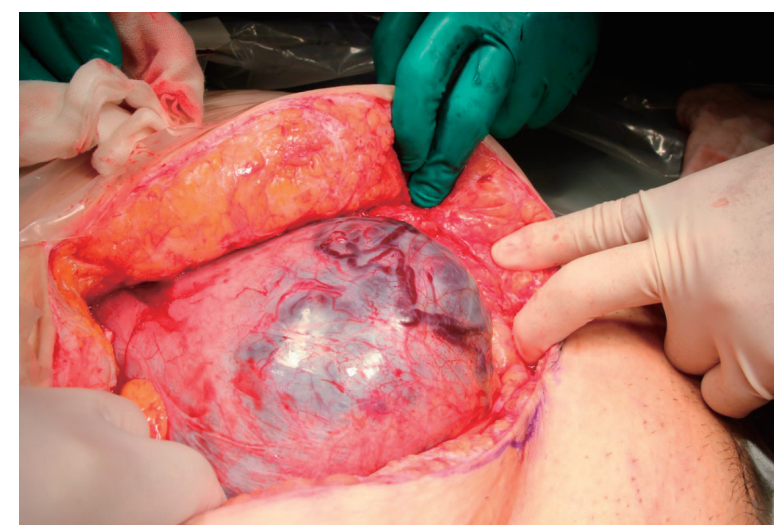

Fig. 2. Image taken during operation. Medusa head infiltration of the uterine surface by the placenta is observed, together with bulging of the anterior uterine wall. was immediately conducted, with no attempt at placenta removal. The total blood loss was $1,370 \mathrm{~mL}$, but maternal condition remained stable and no blood transfusion was required. The patient was discharged in good condition 8 days after the operation. Pathological examination showed villous tissue directly attached to the thin uterine wall, indicating abnormal placental invasion. There was no evidence of villous tissue invasion in the myometrium or uterine serosa (Fig. 3). Later, we obtained the patient's 9 -week ultrasound findings from the hospital she previously visited. These showed an empty uterine cavity and a gestational sac with fetal heartbeat in her cesarean scar (Fig. 4). Our final diagnosis was unexpected PAS caused by a late diagnosed CSP.
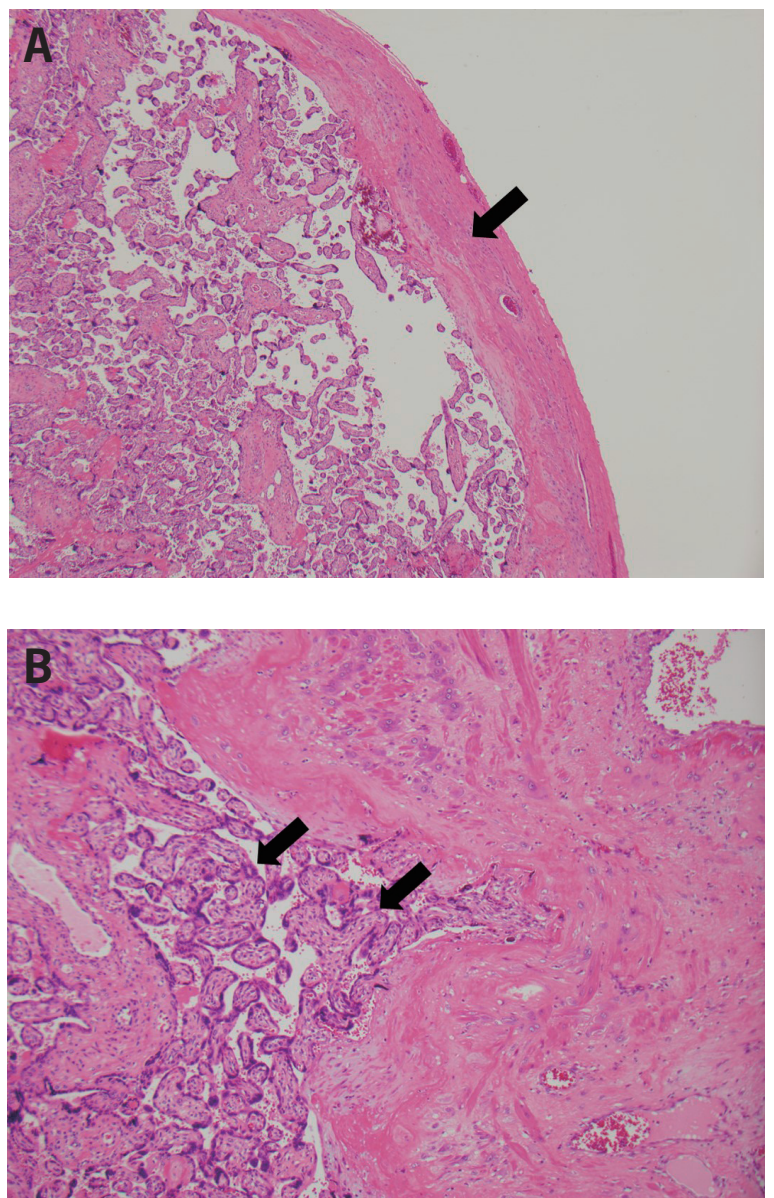

Fig. 3. Histologic image of the resected uterine wall at the site of placental attachment. (A) The myometrium (arrow) maintains its original formation, although it is extremely thin, indicating CSP (hematoxylin-eosin stain, original magnification $\times 4$ ). (B) Villous tissue (arrows) directly attached to the thin uterine wall without decidual tissue. Note no evidence of villous tissue invasion in the myometrium or uterine serosa. Placenta accreta was diagnosed (hematoxylin-eosin stain, original magnification $\times 10$ ). 


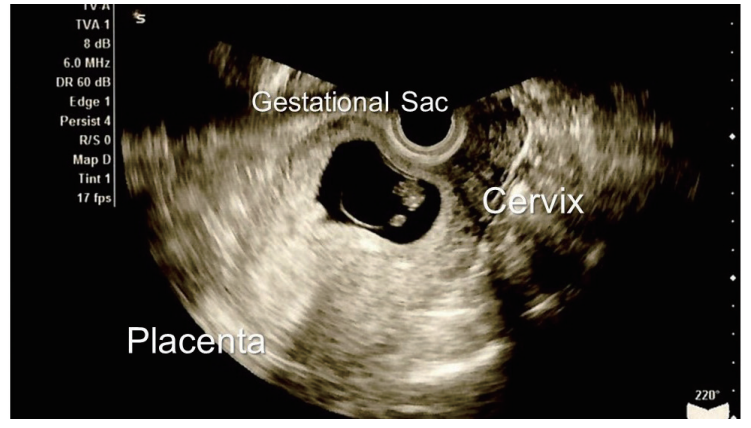

Fig. 4. Transvaginal ultrasound at 9 weeks performed at another hospital, indicating likely cesarean scar pregnancy. Note the gestational sac at the site of a previous cesarean scar.

\section{Discussion}

CSP is a rare form of ectopic pregnancy, accounting for $6.1 \%$ of ectopic pregnancies and $0.15 \%$ of pregnancies in women who have previously undergone $\mathrm{CS}^{3,4)}$. In most cases, CSP is diagnosed between 6 and 8 weeks of gestation because this is when the gestational sac is most clearly visible on ultrasound $^{5)}$. Because CSP is rare, its obstetric outcome with expectant management is not well known.

Recently, Cali et al..$^{6)}$ conducted a systematic review and meta-analysis of 52 expectantly managed cases of CSP. In $9.9 \%$ of cases (95\% confidence interval $[\mathrm{CI}], 2.9-20.4 \%)$, uterine rupture occurred during the first or second trimester, and hysterectomy was required in $15.2 \%$ of cases $(95 \%$ CI, 3.6$32.8 \%)$. A total of 40 cases $(76.9 \%$ [95\% CI, 65.4$68.5 \%$ ]) progressed to the third trimester of pregnancy ; in $74.8 \%$ of these (95\% CI, 52.0 $92.1 \%$ ), PAS was diagnosed clinically or pathologically, with $69.7 \%$ of PAS cases (95\% CI, 42.8-90.1\%) being placenta percreta. These results suggest that CSP is a risk factor for PAS ${ }^{1,6)}$.

PAS encompasses various degrees of pathologic adherence of the placenta, including placenta increta, placenta percreta, and placenta accreta. The most favored hypothesis regarding the etiology of PAS is the placenta adhering to a defective site in the decidua $^{7)}$. Placenta previa in a patient with a history of CS is a strong risk factor for PAS. In such cases, careful diagnosis and multidisciplinary management strategies are required to reduce the risk of morbidity before $\mathrm{CS}^{8)}$. However, on occasion, PAS may be diagnosed for the first time at the time of delivery. Kyozuka et $a l{ }^{9}{ }^{9}$ recently reported that undergoing CS two or more times, smoking during pregnancy, becoming pregnant through assisted reproductive technology, and uterine anomalies are all independent risk factors for unexpected PAS in the absence of placenta previa. Therefore, it is crucial to maintain a high index of suspicion for PAS during the antenatal period in patients with these risk factors, even without placenta previa. Many such patients have been clinically diagnosed with PAS, and these situations may require unexpected medical intervention, including manual removal of the adherent placenta, which can cause life-threatening postpartum hemorrhage ${ }^{10)}$ and/or increased morbidity ${ }^{11)}$.

CSPs are divided into two types based on imaging presentation and progression ${ }^{12)}$. In type 1 (endogenic CSP), the gestational sac grows toward the uterine cavity, and in type 2 (exogenic CSP), the gestational sac grows toward the bladder. In this case, although the gestational sac was observed in the area of the cesarean scar at 9 weeks, the diagnosis of CSP was delayed, and emergent cesarean hysterectomy was required because of unexpected PAS. We conclude that the present case was a type 1 CSP in which the placenta and fetus had already grown toward the uterine cavity, resulting in the absence of placenta previa and underestimation of the risk of PAS.

Our experience shows that PAS can occur in the absence of placenta previa as a result of type 1 CSP. As the incidence of CSP appears to be rising $^{13)}$, type $1 \mathrm{CSP}$, in particular, may be increasing. Therefore, early diagnosis of CSP (between 6 and 8 weeks) is essential to ensure a high index of suspicion for unexpected PAS. It is important to suspect PAS if the placenta is present around a cesarean scar in the second to third trimester. This report illustrates the importance of ultrasound during early gestation in preventing the delayed diagnosis of CSP, which may result in unexpected PAS necessitating emergent hysterectomy.

\section{Contributors}

All authors were involved in the clinical care of the patient and contributed to the conception, drafting, review and revision of the manuscript. All authors saw and approved the final version of the paper and take full responsibility for the work.

\section{Funding}

This work did not receive any specific grant from funding agencies. 


\section{Patient Consent}

Informed consent was obtained from the patients for publication of this work.

\section{Provenance and Peer Review}

This case report was peer reviewed.

\section{Declaration of Competing Interest}

The authors have no potential conflicts of interest to declare.

\section{References}

1. Timor-Tritsch IE, Monteagudo A, Cali GA, et al. Cesarean scar pregnancy is a precursor of morbidly adherent placenta, Ultrasound Obstet. Gynecol, 44 : 346-353, 2014.

2. Silver RM, Landon MB, Rouse DJ, et al. National Institute of Child Health and Human Development Maternal-Fetal Medicine Units Network, Maternal morbidity associated with multiple repeat cesarean deliveries. Obstet Gynecol, 107 : 1226-1232, 2006.

3. Tamada S, Masuyama H, Maki J, et al. Successful pregnancy located in a uterine cesarean scar : a case report. Case Rep Womens Health, 14: 810, 2017.

4. Seow KM, Huang LW, Lin YH, Lin MY, et al. Cesarean scar pregnancy : issues in management, Ultrasound Obstet. Gynecol, 23 : 247-253, 2004.

5. Agten AK, Cali G, Monteagudo A, et al. The clini- cal outcome of cesarean scar pregnancies implanted "on the scar" versus "in the niche." Am J Obstet Gynecol, 216 : 510.e1-e6, 2017.

6. Cali G, Timor-Tritsch IE, Jaraquemada J, et al. Outcome of Cesarean scar pregnancy managed expectantly : systematic review and meta-analysis. Ultrasound Obstet Gynecol, 51 : 169-175, 2018.

7. Oyelese Y, Smulian JC. Placenta previa, placenta accreta, and vasa previa. Obstet Gynecol, 107 : 927-941, 2006.

8. Shamshirsaz AA, Fox KA, Salmanian B, et al. Maternal morbidity in patients with morbidly adherent placenta treated with and without a standardized multidisciplinary approach. Am J Obstet Gynecol, 212 : 218.e1-e9, 2015.

9. Kyozuka H, Yamaguchi A, Suzuki D, et al. Risk factors for placenta accreta spectrum : findings from the Japan Environment and Children's Study. BMC Pregnancy Childbirth, 19 : 447, 2019.

10. Eller AG, Porter TF, Soisson P, et al. Optimal management strategies for placenta accreta. BJOG, 116 : 648-654, 2009.

11. Yap YY, Perrin LC, Pain SR, et al. Manual removal of suspected placenta accreta at cesarean hysterectomy. Int J Gynaecol Obstet, 100 : 186-187, 2008.

12. Jo EJ, Cha HH, Seong WJ. Delayed diagnosis of a cesarean scar pregnancy : a case report. J Med Case Rep, 13 : 53, 2019.

13. Timor-Tritsch IE, Monteagudo A. Unforeseen consequences of the increasing rate of cesarean deliveries : early placenta accreta and cesarean scar pregnancy. A review. Am J Obstet Gynecol, 207 : 14-29, 2012. 